\title{
Nobiletin alleviates ischemia/reperfusion injury in the kidney by activating PI3K/AKT pathway
}

\author{
BO LIU, QUANHONG DENG, LEI ZHANG and WEN ZHU \\ Department of Urology, Jingmen No. 2 People's Hospital, Jingmen, Hubei 448000, P.R. China
}

Received February 13, 2020; Accepted September 15, 2020

DOI: $10.3892 / \mathrm{mmr} .2020 .11554$

\begin{abstract}
Recent studies have demonstrated that nobiletin (NOB) displays anti-oxidative and anti-apoptotic efficacies against multiple pathological insults. However, the potential effects of NOB on the injury caused by ischemia and reperfusion $(I / R)$ in the kidney remain undetermined. In the present study, I/R injury was elicited by right kidney removal and left renal pedicel clamping for $45 \mathrm{~min}$, followed by reperfusion for $24 \mathrm{~h}$. NOB was added at the start of reperfusion. Histological examination, detection of biomarkers in plasma, and measurement of apoptosis induced by endoplasmic reticulum stress (ERS) were used to evaluate renal injury. Additionally, the PI3K/AKT inhibitor LY294002 was also used in mechanistic experiments. NOB pre-treatment significantly reduced renal damage caused by I/R injury, as indicated by decreased serum levels of creatine, blood urea nitrogen and tubular injury scores. Furthermore, NOB inhibited elevated ERS-associated apoptosis, as evidenced by reduced apoptotic rates and ERS-related signaling molecules (such as, C/EBP homologous protein, caspase-12 and glucose-regulated protein of $78 \mathrm{kDa}$ ). NOB increased phosphorylation of proteins in the PI3K/AKT pathway. The inhibition of PI3K/AKT signaling with pharmacological inhibitors could reverse the beneficial effects of NOB during renal I/R insult. In conclusion, NOB pre-treatment may alleviate I/R injury in the kidney by inhibiting reactive oxygen species production and ERS-induced apoptosis, partly through the PI3K/AKT signaling pathway.
\end{abstract}

\section{Introduction}

Ischemia/reperfusion (I/R) injury in the kidney is a severe clinical condition associated with acute inflammation and progressive deterioration of renal function (1). However, the mechanisms underlying renal $I / R$ injury remain unclear.

Correspondence to: Dr Wen Zhu, Department of Urology, Jingmen No. 2 People's Hospital, 39 Xiangshan Avenue, Jingmen, Hubei 448000, P.R. China

E-mail: zhuwenww@163.com

Key words: renal ischemia, reperfusion, nobiletin, PI3K, AKT, apoptosis
Previous studies have demonstrated that oxidative stress, apoptosis, and inflammation are involved in I/R injury of the kidney (2-5). To develop effective prevention measures, it is necessary to understand the pathological mechanisms of renal I/R damage. Thus, the present study aimed to examine potential mechanisms that could prevent renal I/R injury.

Nobiletin (NOB; 3',4',5,6,7,8-hexamethoxyflavone) is a dietary polymethoxylated flavonoid isolated from citrus fruits with protective effects on I/R injury (6-8). Liu et al (6) indicated that NOB could attenuate I/R injury in H9c2 cardiomyocytes by activating the Akt/GSK-3 $\beta$ signaling pathway. Zheng et al (8) suggested that NOB protected cerebral neurons from I/R-induced injury through the MAPK pathway. Nevertheless, the exact roles of NOB in I/R injury of the kidney remain to be determined.

Endoplasmic reticulum stress (ERS) is considered a driving force of acute renal failure (ARF) induced by I/R (9). Increasing evidence indicates that persistent activation of ERS and subsequent elevation of apoptotic cascades are key to the pathogenesis of renal I/R injury (10-13). Additionally, a previous study confirmed that apoptosis occurs in the early phase of ischemia and can be worsened by reperfusion (13). Although the benefits of inhibition of ERS-induced apoptosis against renal I/R injury are well-known, the potential mechanisms of ERS-related apoptosis still remain to be investigated (14). Moreover, therapeutic approaches targeting ERS-related renal apoptosis remain to be studied.

The PI3K/AKT pathway affects several biological processes, including cellular proliferation and organ growth (15-17). Recent evidence demonstrates that the PI3K/AKT pathway can ameliorate I/R injury in the kidney (18), liver (19), myocardium (20) and brain $(21,22)$. Wei et al (23) suggested that propofol could attenuate renal I/R injury by modulating PI3K/AKT signaling and partially reduce apoptosis and secretion of pro-inflammatory cytokines (23).

The present study aimed to determine whether NOB pre-treatment could attenuate renal $\mathrm{I} / \mathrm{R}$ injury in vivo and to verify the hypothesis that the protective effects of NOB are related to activation of the PI3K/AKT pathway.

\section{Materials and methods}

Experimental animals. A total of $30 \mathrm{C} 57 \mathrm{BL} 6 / \mathrm{J}$ male mice aged 6-8 weeks (15-20 g) were provided by Vital River Laboratories. All animals were housed and maintained in a 
temperature- $\left(20 \pm 5^{\circ} \mathrm{C}\right)$ and a humidity-controlled $(40-70 \%)$ animal facility adhering to a $12-\mathrm{h}$ light/dark cycle, in nondirectional airflow cages. Mice were provided with water and rodent chow ad libitum. The present study was approved by Jingmen No. 2 People's Hospital Laboratory Animals Ethics Committee (approval no. 4237668). All experiments adhered to the Guide for the Care and Use of Laboratory Animals published by the US National Institutes of Health (the 8th Edition, NRC 2011).

Renal I/R model and treatment. NOB was obtained from Sigma-Aldrich (Merck KGaA; purity, >99\%). All mice were anesthetized with intraperitoneal (i.p.) injection of $40 \mathrm{mg} / \mathrm{kg}$ pentobarbital sodium. Laparotomy followed by nephrectomy on the right side were then performed. Subsequently, the left kidney was clamped by a non-traumatic vessel forceps for $45 \mathrm{~min}$ to establish a model of ischemia, followed by reperfusion for $24 \mathrm{~h}$. A total of 30 mice were divided into six groups ( $n=5$ in each group). Except for two mice that died of unexplained death after I/R injury surgery, all mice were sacrificed by cervical dislocation following anesthesia with $40 \mathrm{mg} / \mathrm{kg}$ i.p. pentobarbital sodium. The groups were as follows: i) in the sham group, the right kidney was removed; ii) in the I/R group, a vessel forceps was used to occlude the vessels of the left kidney for $45 \mathrm{~min}$, followed by reperfusion for $24 \mathrm{~h}$; ii) in the I/R + NOB groups, mice received NOB $(25,50$, or $100 \mathrm{mg} / \mathrm{kg}$ ) i.p. injection for 7 consecutive days, followed by the same surgical procedures as the $I / R$ group. The duration of the experiment was 9 days.

Evaluation of the kidney function. Renal function was evaluated by determination of blood urea nitrogen (BUN) and serum creatinine $(\mathrm{Cr})$ levels. At the 24 th $\mathrm{h}$ post reperfusion, blood was taken from the tail vein and centrifuged to collect serum. BUN and SCr were measured using ELISA kits (cat. nos. SJH-033595 and C011-1-1; T\&T Scientific Corporation).

Evaluation of renal injury. The morphological changes of renal tubules were observed, and the degree of renal injury was determined using hematoxylin and eosin $\mathrm{H} \& \mathrm{E}$ staining. The kidney was isolated, washed, fixed in $4 \%$ paraformaldehyde and embedded with paraffin. The samples were cut into sections with a thickness of $3 \mu \mathrm{m}$. After dewaxing with xylene, the sections were sequentially incubated for $5 \mathrm{~min}$ in water and alcohol of different concentrations. H\&E staining was then carried out at room temperature with hematoxylin dye solution for $5 \mathrm{~min}$, followed by rinsing with tap water/for $5 \mathrm{~min}$ and incubation with eosin solution for $2 \mathrm{~min}$. Sections were observed under a light microscope (magnification, $\mathrm{x} 400$ ). Quantitative analysis was conducted using Image-Pro Plus (version 6.0; National Institutes of Health) to assess capsule area, tubular injury scores, and glomerular tuft area as mentioned previously (24).

Detection of apoptosis by flow cytometry. Single-cell suspensions were prepared from fresh tissue. The cells were then washed twice with PBS, centrifuged $\left(4^{\circ} \mathrm{C}, 1,000 \mathrm{xg}, 5 \mathrm{~min}\right)$ and collected. Subsequently, the cells were resuspended in $500 \mu 1$ binding buffer, then with $10 \mu 1$ Annexin V-FITC and $5 \mu \mathrm{l}$ PI. The samples were then incubated at room temperature for 5 min in the dark, then acquired on a BD FACSCanto flow cytometer (BD Biosciences). The flow cytometry data was analyzed using BD FACSDiva (BD Biosiences). In the flow cytometry dotplots, the Annexin $\mathrm{V}^{+} \mathrm{PI}^{+}$quadrant represents cells in late apoptosis, while the Annexin $\mathrm{V}^{+} \mathrm{PI}^{-}$quadrant shows cells in early apoptosis.

Reverse transcription-quantitative (RT-q) PCR. mRNA levels were determined by RT-qPCR, as previously described $(3,16)$. Extraction of total RNA from the animals kidney was carried out using TRIzol ${ }^{\circledR}$ (Invitrogen; Thermo Fisher Scientific, Inc.) after the indicated treatments. cDNA was obtained from mRNA by reverse transcription (at $42^{\circ} \mathrm{C}$ for $60 \mathrm{~min}, 70^{\circ} \mathrm{C}$ for $5 \mathrm{~min}$ and $4^{\circ} \mathrm{C}$ for $15 \mathrm{~min}$ preservation) using a commercial Reverse Transcription cDNA Synthesis kit (Thermo Fisher Scientific, Inc.). RT-qPCR was conducted with SYBR-Green Master Mix kit (Thermo Fisher Scientific, Inc.) using the 7500 system ABI Prism system (Thermo Fisher Scientific, Inc.). The thermocycling conditions were as follows: i) $2 \mathrm{~min}$ at $50^{\circ} \mathrm{C}$; ii) $95^{\circ} \mathrm{C}$ for $10 \mathrm{~min}$; and iii) 40 cycles at $95^{\circ} \mathrm{C}$ for $30 \mathrm{sec}$, and $60^{\circ} \mathrm{C}$ for $30 \mathrm{sec}$. The mRNA expression levels of C/EBP homologous protein (CHOP), glucose-regulated protein of $78 \mathrm{kDa}$ (GRP78), and caspase-12 were normalized to those of GAPDH.

The following primers were used: i) CHOP-reverse, 5'-CTT CAGCAAGCTGTGCCACT-3'; ii) CHOP-forward, 5'-TAGCTT GGCTGACTGAGGAGC-3'; iii) GRP78-reverse, 5'-GCAAAC TTCTCGGCGTCATT-3'; iv) GRP78-forward, 5'-GATAAT CAGCCCACCGTAACAAT-3'; v) caspase-12-reverse, 5'-CCT TCCTTCTCCATCACTGGA-3'; vi) caspase-12-forward, 5'-CATTGCCAATTCCGACAAAC-3'; vii) GAPDH-reverse, 5'-TTTGAGGGTGCAGCGAACTT-3'; and viii) GAPDHforward, 5'-ACAGCAACAGGGTGGTGGAC-3'.

Western blot analysis. Renal samples were homogenized on ice with an IKA homogenizer $\left(12,000 \mathrm{x} \mathrm{g} ; 15 \mathrm{~min}\right.$ at $\left.4^{\circ} \mathrm{C}\right)$, then lysed in RIPA lysis buffer [50 mM Tris- $\mathrm{HCl}, \mathrm{pH} 7.5$, $150 \mathrm{mM} \mathrm{NaCl}, 1 \% \mathrm{NP}-40$ (v/v), $1 \mathrm{mg} / \mathrm{ml} \mathrm{SDS}, 5 \mathrm{mg} / \mathrm{ml}$ hyodeoxycholic acid sodium, $1 \mathrm{mM}$ PMSF, $10 \mathrm{mg} / \mathrm{ml}$ aprotinin, $10 \mathrm{mg} / \mathrm{ml}$ leupeptin and $10 \mathrm{mg} / \mathrm{ml}$ pepstatin A]. SDS-PAGE (10\%) was used to separate the proteins $(\sim 40 \mu \mathrm{g})$, which were then transferred onto polyvinylidene fluoride membranes (Bio-Rad Laboratories, Inc.). The membranes were blocked with 5\% non-fat milk for $2 \mathrm{~h}$ at room temperature. Protein strips were then incubated with a primary antibody overnight at $4^{\circ} \mathrm{C}$ and then washed with 5\% bovine serum albumin (BSA; Gibco; Thermo Fisher Scientific, Inc.) in PBS/0.1\% Tween-20 and incubated with the secondary antibody for $2 \mathrm{~h}$ at room temperature. The protein strip was developed with a EZ-ECL kit (Biological Industries) and the protein quantity was analyzed using ImageJ software (version 6.0; National Institutes of Health). GAPDH was used as an endogenous control. The primary antibodies used in the study were as follows: anti-GAPDH (mouse; 1:1,000; cat. no. sc-47724; Santa Cruz Biotechnology); anti-AKT (rabbit; 1:500; cat. no. 9139; Cell Signaling Technology); anti-phosphorylated (p)-AKT (rabbit; 1:1,000; cat. no. 9145; Cell Signaling Technology); anti-PI3K (rabbit; 1:800; cat. no. 3230S; Cell Signaling Technology); anti-p-PI3K (rabbit; 1:600; cat. no. 3776S; Cell Signaling Technology). The secondary antibodies included 

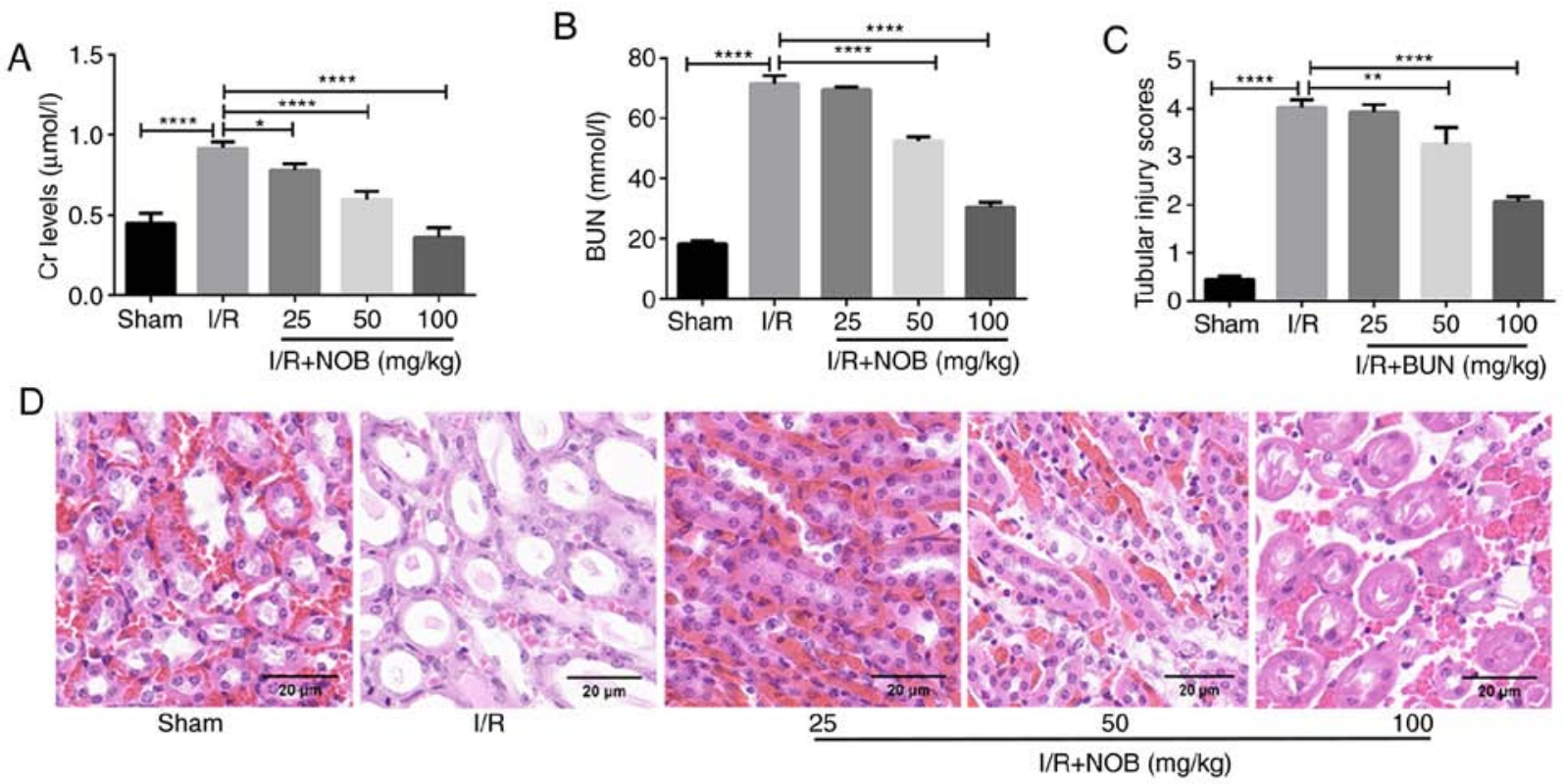

Figure 1. NOB pre-treatment reduces renal damage. (A) Cr levels in serum ( $\mu \mathrm{mol} / \mathrm{l})$. (B) BUN levels in serum (mmol/l). (C) Tubular injury scores. (D) Renal tissue H\&E staining. Magnification, $\mathrm{x} 200$. Scale bar, $20 \mu \mathrm{m} . \mathrm{n}=3$. ${ }^{*} \mathrm{P}<0.05,{ }^{* *} \mathrm{P}<0.01,{ }^{* * * * *} \mathrm{P}<0.0001$. NOB, nobiletin; Cr, creatinine; $\mathrm{I} / \mathrm{R}$, ischemia/reperfusion; BUN, blood urea nitrogen; CHOP, C/EBP homologous protein.

horseradish peroxidase (HRP)-conjugated goat anti-mouse IgG (1:3,000; cat. no. SA00001-1; ProteinTech Group, Inc.) and HRP-conjugated goat anti-rabbit $\operatorname{IgG}(1: 3,000$; cat. no. SA00001-2; ProteinTech Group, Inc.).

Statistical analysis. SPSS 22.0 (IBM Corp.) was used for statistical analysis. All data are presented as the mean \pm SD. The experiments were repeated three times. Multiple comparisons was performed with one-way ANOVA followed by Tukey's post hoc test. Paired student's t-test was performed for comparisons between groups. $\mathrm{P}<0.05$ was considered to indicate a statistically significant difference.

\section{Results}

NOB pre-treatment reduces renal damage caused by I/R injury. To investigate the role of NOB in renal damage, an in vivo model of renal I/R was established. The levels of $\mathrm{Cr}$ and BUN were measured as markers of renal injury. Compared with the sham group, the levels of $\mathrm{Cr}$ and BUN were significantly increased in the $\mathrm{I} / \mathrm{R}$ group, indicating that the kidney suffered damage following I/R injury. In contrast, $\mathrm{Cr}$ and BUN levels after NOB treatment $(25,50$ and $100 \mathrm{mg} / \mathrm{kg})$ were significantly decreased, compared with the I/R group. NOB decreased $\mathrm{Cr}$ and BUN levels in a dose-dependent manner (Fig. 1A and B). In addition, NOB concentrations significantly reduced BUN levels starting at $50 \mathrm{mg} / \mathrm{kg}$, compared with the I/R group.

$\mathrm{H} \& \mathrm{E}$ staining was performed using kidney tissues from each group, and renal tubular damage was observed (Fig. 1D). In comparison with the sham group, renal tubular injury was most apparent in the I/R group. Renal tubular injury was attenuated after different doses of NOB treatment. Tubular injury scores we also evaluated in order to obtain more objective and reliable assessment of renal injury (Fig. 1C). A concentration of $25 \mathrm{mg} / \mathrm{kg}$ NOB did not relieve renal tubular damage.
Nevertheless, NOB concentrations $\geq 50 \mathrm{mg} / \mathrm{kg}$ significantly reduced tubular injury scores, compared with the I/R group. These results indicated that NOB could reduce $\mathrm{Cr}$ and BUN levels, as well as renal damage following $\mathrm{I} / \mathrm{R}$ injury.

NOB suppresses ERS-related apoptosis in renal I/R injury. I/R kidney injury is closely related ERS-induced apoptosis (25), and NOB is reported to be involved in the regulation of ERS (26). GRP78 is an ER chaperone that plays an essential role in apoptosis during renal I/R injury (27). Additionally, ERS contributes to apoptosis through CHOP-dependent signaling pathways (28). To explore whether the protective effects of NOB on renal I/R injury are associated with ERS, flow cytometry was used to evaluate apoptosis. Moreover, mRNA expression levels of the ERS-related markers GRP78, CHOP, and caspase-12 were determined by RT-qPCR. GRP78, CHOP, and caspase-12 mRNA levels in the I/R group significantly increased, in comparison with the sham group. By contrast, NOB could mitigate ERS by significantly decreasing the mRNA expression levels of these molecules, compared with the I/R group (Fig. 2A-C).

Flow cytometry results are shown in Fig. 2D. Early apoptosis and late apoptosis were increased in the I/R group, compared with the control group. Late apoptosis gradually decreased with increasing NOB concentrations. Moreover, while $25 \mathrm{mg} / \mathrm{kg}$ NOB did not markedly inhibit the increase of early apoptosis caused by I/R, when NOB concentrations reached $50 \mathrm{mg} / \mathrm{kg}$, early apoptosis rates decreased. Thus, NOB suppressed ERS induced by I/R and apoptosis in kidney. Based on these results, $50 \mathrm{mg} / \mathrm{kg} \mathrm{NOB}$ was used in all subsequent experiments.

$N O B$ promotes the PI3K/AKT pathway in renal I/R injury. The PI3K/AKT pathway is known to promote survival and reduce apoptosis following renal I/R injury (29). In order 
A

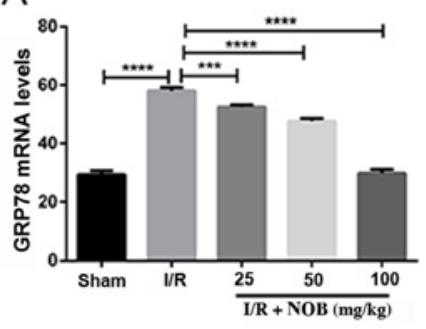

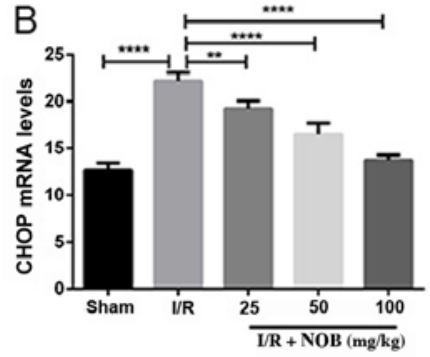

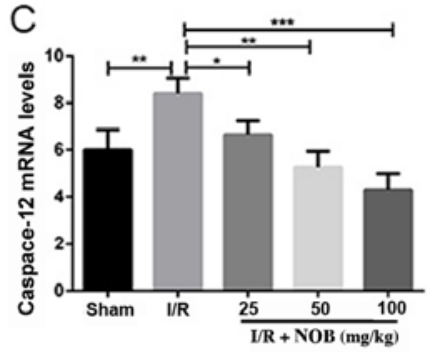

D
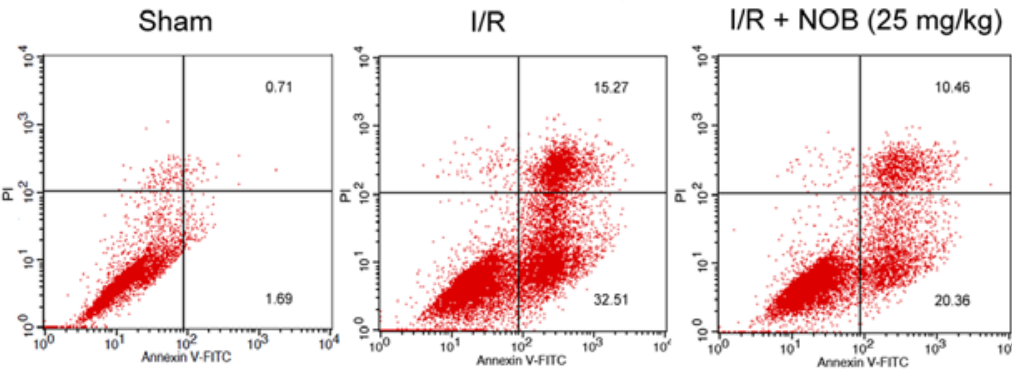

$\mathrm{l} / \mathrm{R}+\mathrm{NOB}(50 \mathrm{mg} / \mathrm{kg})$

$\mathrm{l} / \mathrm{R}+\mathrm{NOB}(100 \mathrm{mg} / \mathrm{kg})$
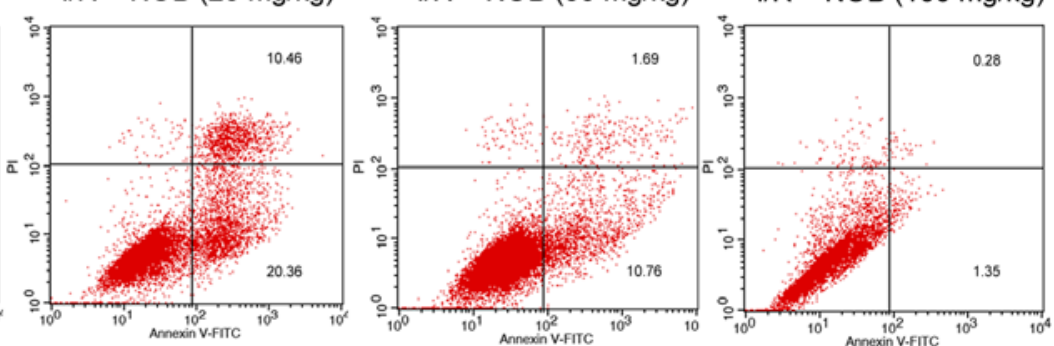

Figure 2. NOB inhibits ERS-associated apoptosis. (A) GRP78 mRNA levels. (B) CHOP mRNA levels. (C) Caspase-12 mRNA levels. n=3. ${ }^{*} \mathrm{P}<0.05$, ${ }^{* *} \mathrm{P}<0.01$, ${ }^{* * * *} \mathrm{P}<0.001,{ }^{* * * *} \mathrm{P}<0.0001$. (D) Flow cytometry pattern for apoptosis. Annexin $\mathrm{V}^{+} \mathrm{PI}^{+}$and Annexin $\mathrm{V}^{+} \mathrm{PI}$ cells were considered apoptotic (late apoptotic and early apoptotic, respectively). NOB, nobiletin; ERS, endoplasmic reticulum stress; I/R, ischemia/reperfusion; CHOP, C/EBP homologous protein; FITC, fluorescein isothiocyanate; PI, propidium iodide.

A
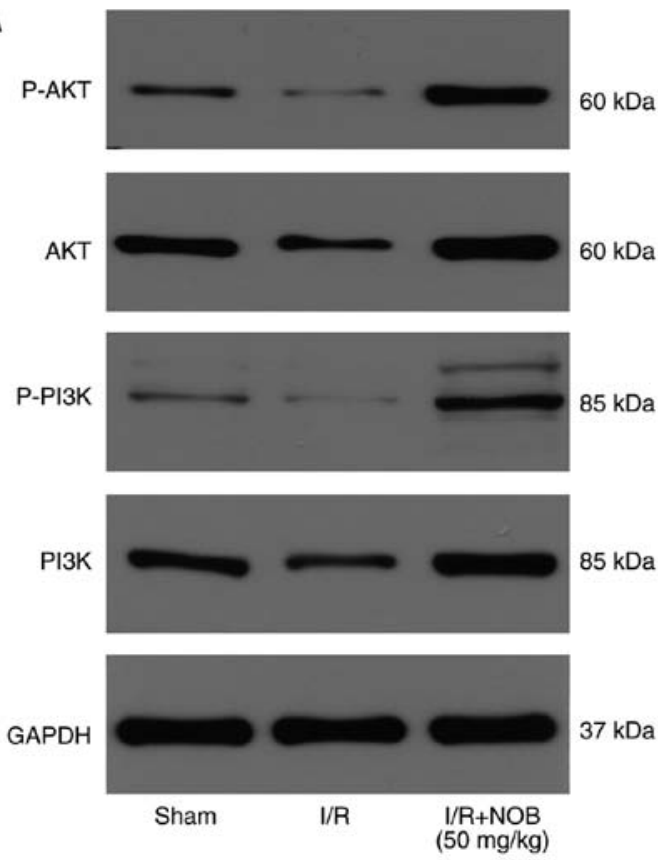

B

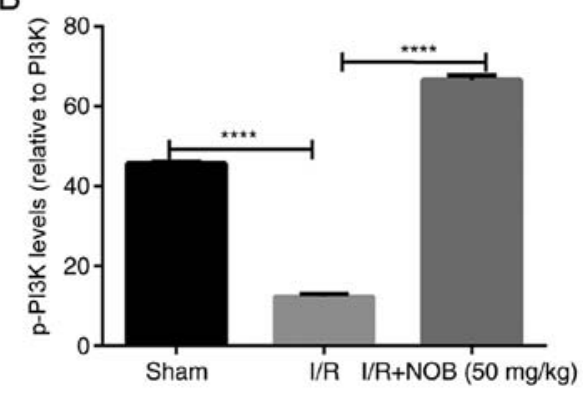

C

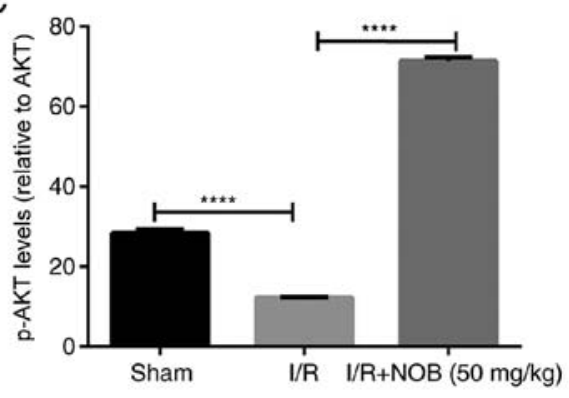

Figure 3. NOB activates the PI3K/AKT signaling pathway. (A) p-AKT/AKT, p-PI3K/PI3K protein levels. (B and C) Grayscale analysis of protein expression. $\mathrm{n}=3 .{ }^{* * * *} \mathrm{P}<0.0001 \mathrm{NOB}$, nobiletin; I/R, ischemia/reperfusion; $\mathrm{p}$, phosphorylated.

to determine if the PI3K/AKT pathway participates in the amelioration of renal I/R injury mediated by NOB, the protein levels of molecules involved in the PI3K/AKT pathway were measured by western blot analysis (Fig. 3). The levels of phosphorylated AKT and PI3K were significantly decreased in the I/R group, compared with the sham group. Notably, p-PI3K/AKT levels were significantly increased following NOB treatment, compared with the I/R group. Therefore, NOB could ameliorate renal I/R injury by activating the PI3K/AKT pathway.
Inhibition of PI3K/AKT reverses the effects of $N O B$ on $I / R$ injury and increases the levels of ERS-associated markers following renal $I / R$ insult. In order to further examine the role of the PI3K/AKT in the amelioration of renal I/R injury, mice were treated with LY294002, a specific PI3K/AKT inhibitor, following renal I/R injury. The effect of NOB on kidney damage were inhibited following treatment with LY294002, as indicated by elevated levels of $\mathrm{Cr}$ and BUN, as well as GRP78, CHOP, and caspase-12 expression (Fig. 4A-F), and enhanced tubular injury (Fig. 4C and G) in the I/R + NOB + LY294002 

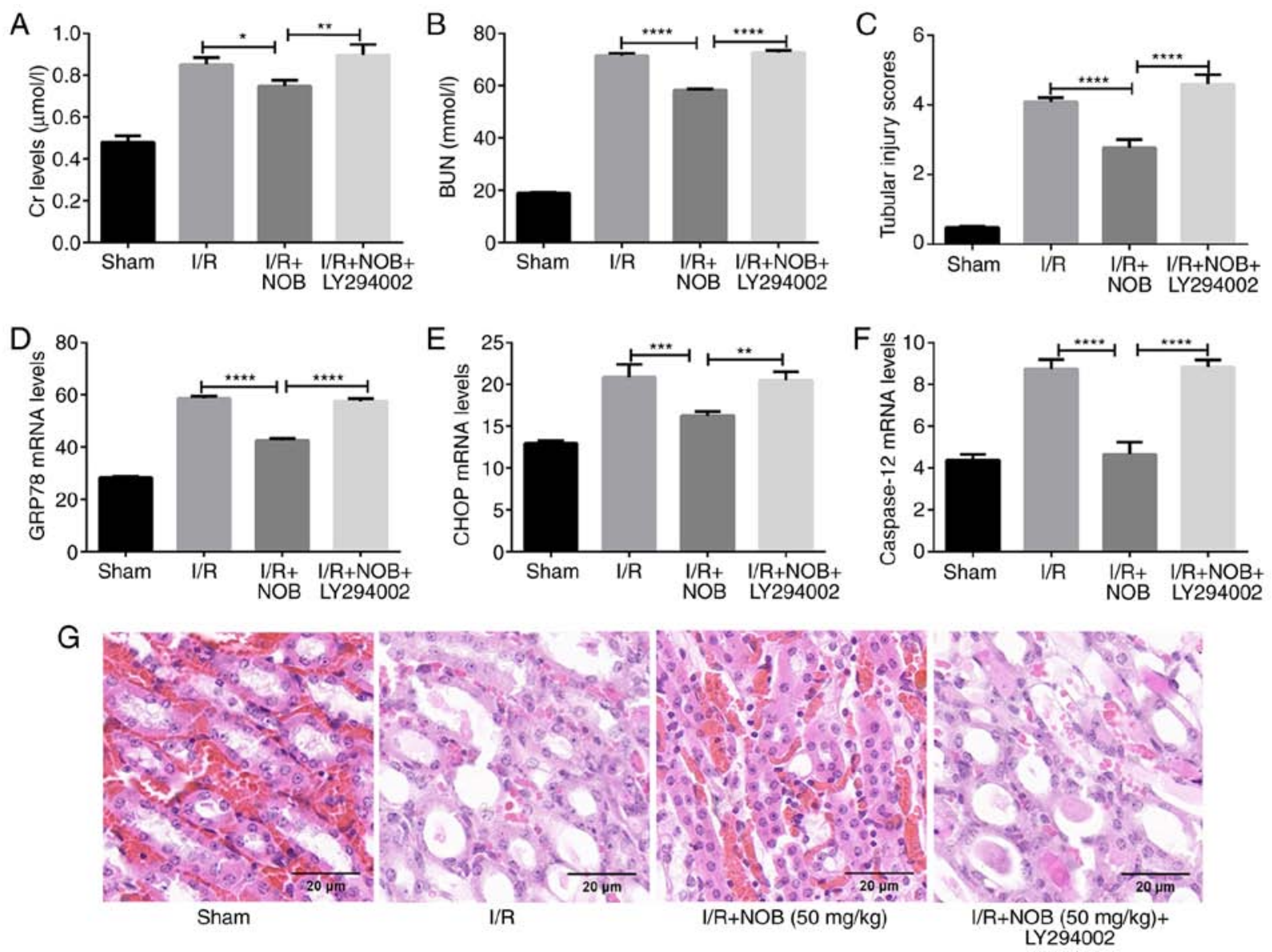

Figure 4. inhibition of the PI3K/AKT pathway reverses the effects of NOB. (A) Cr levels in serum ( $\mu \mathrm{mol} / \mathrm{l})$. (B) BUN levels in serum (mmol/l). (C) Tubular injury scores. (D) GRP78 mRNA levels. (E) CHOP mRNA levels. (F) Capase-12 mRNA levels. (G) H\&E staining of renal tissues. Magnification, x200. Scale bar, $20 \mu \mathrm{m} . \mathrm{n}=3 .{ }^{*} \mathrm{P}<0.05,{ }^{* *} \mathrm{P}<0.01,{ }^{* * * *} \mathrm{P}<0.001,{ }^{* * * *} \mathrm{P}<0.0001$. NOB, nobiletin; Cr, creatinine; $\mathrm{I} / \mathrm{R}$, ischemia/reperfusion; BUN, blood urea nitrogen; CHOP, C/EBP homologous protein.

group, compared with the I/R + NOB group. Thus, NOB could ameliorate kidney damage caused by ERS in renal I/R injury by activating the PI3K/AKT pathway.

\section{Discussion}

Renal I/R injury is one of the leading causes of acute kidney injury and has a high mortality rate (30). In previous studies, it was proposed that the anti-apoptotic, antioxidant, and anti-inflammatory properties of NOB could protect against I/R insult $(7,10)$. In the present study, NOB could relieve I/R injury in the kidney. The levels of BUN and serum $\mathrm{Cr}$ were used as indicators of renal injury (31). Compared with the sham group, serum $\mathrm{Cr}$ and BUN levels were increased in the I/R group, indicating that renal function was critically impaired. In addition, NOB significantly alleviated renal apoptosis and inhibited protein expression of ERS-related molecules, such as caspase-12, CHOP and GRP78. Furthermore, using a specific PI3K/AKT inhibitor, it was demonstrated that NOB could exert a protective effect by activating PI3K/AKT signaling. These findings indicated that NOB could ameliorate I/R-induced and ERS-related apoptosis mainly through the PI3K/AKT-dependent pathway.

Ample evidence has suggested that NOB appears to be beneficial in kidney disease. Wei et al (32) suggested that NOB elicited cell cycle arrest at the $\mathrm{G}_{0} / \mathrm{G}_{1}$ phase, which suppressed renal cancer cell proliferation and increased cellular apoptosis. Malik et al (33) demonstrated that NOB pretreatment could preserve kidney function and reduce oxidative stress by inhibiting DNA damage and the activation of apoptosis-related pathways. Furthermore, NOB ccould also attenuate tubular injury as seen in histological examination (33). However, whether NOB is involved in renal I/R injury remains unknown, and the underlying mechanisms are largely unclear. Recently, accumulating evidence has suggested that NOB is an important mediator in the initiation and development of ERS and apoptotis (34). Moreover, NOB regulates ERS progression and might be used as a therapeutic strategy to inhibit ERS-related renal apoptosis and cell death (35). Thus, we hypothesize that NOB could exert protective effects on renal tubules and ameliorate ERS-related apoptosis in renal $\mathrm{I} / \mathrm{R}$ injury. The present study provides evidence that NOB has protective effects on renal I/R injury.

It is well-known that the most effective therapy for acute kidney injury is to successfully restore reperfusion in the ischemic region in an early stage $(26,36,37)$. Nevertheless, the accompanied I/R injury largely decreases the benefits of this approach. Although an increasing number of studies have attached importance to the pathological mechanisms of kidney I/R injury, a satisfactory strategy to avoid the adverse 
effects of reperfusion still remains to be explored. Apoptosis is considered a key mechanism of renal I/R injury, in which ERS-elicited apoptosis cascade played a pivotal role $(38,39)$. It has been demonstrated that I/R injury in the kidney causes severe ERS due to increased GRP78 levels (1). Misfolded and unfolded proteins bind with GRP78 competitively, leading to depolymerization between ER-transmembrane transducers and GRP78 (40).CHOP, a member of the C/EBP family, is a specific transcription factor involved in ERS-induced apoptosis. The levels of CHOP are relatively low under normal conditions, but can be markedly increased following prolonged ERS (26). The results of the present study are consistent with a previous study (14) and indicate that I/R contributes to sustained ERS and severe renal injury, as evidenced the increased levels of caspase-12,CHOP and GRP78, as well as apoptotic rates. However, NOB reduced the expression levels of these proteins and attenuated the severity of I/R injury. Therefore, the protective effects of NOB on the kidney are mediated, at least in part, by the inhibition of ERS and apoptosis.

The potential molecular mechanisms related to the protective roles of NOB against renal I/R injury and ERS-related apoptosis were also examined. PI3K and its downstream target serine/threonine kinase AKT belong to a conserved family of signaling molecules $(26,41,42)$. PI3K/AKT pathway activation is an endogenous regulatory mechanism that promotes cell survival in response to harmful external stimuli. Notably, numerous studies suggest that the PI3K/AKT pathway plays a pivotal role in the pathological process of renal $I / R$ injury, in which inhibition of ERS-related apoptosis is an important mechanism (43,44). Zhang et al (26) have reported that PI3K/AKT signaling inhibits I/R injury by suppressing ERS, thus reducing GRP78, CHOP, and caspase-12 levels and apoptosis. Moreover, NOB can promote the activation of PI3K/AKT signaling pathway $(45,46)$. Li et al $(45)$ suggested that NOB could reduce the apoptosis induced by ERS in during oxygen-glucose deprivation/reoxygenation injury by activating the PI3K/AKT pathway in PC12 cells. Consistent with previous findings, the present study confirmed that pretreatment with NOB inhibited apoptosis resulting from renal I/R injury and upregulated AKT and PI3K phosphorylation. To confirm this, a specific inhibitor of PI3K/AKT signaling, LY294002, was also added at the onset of I/R. The protective effect of NOB and inhibition of ERS-related apoptosis were significantly reversed in the presence of LY294002, confirming that NOB administration could attenuate I/R injury through the PI3K/AKT pathway. However, the relationship between NOB and the PI3K/AKT pathway remain controversial. Indeed, several studies demonstrated that the anticancer effect of NOB was mediated by the PI3K/AKT pathway (46). These studies are inconsistent with a previous report suggesting that NOB markedly represses migration and proliferation of non-renal tubular cells by inhibiting AKT signaling (26). These discrepancies might be partially explained by the fact that the role of NOB in the activation of the PI3K/AKT pathway could be cell- and stimulus-dependent.

One of the limitations of the study is that expression of CHOP, caspase-12 and GRP78 were not detected at the protein level. In addition, one of the classic methods for detecting apoptosis, TUNEL staining is not used, although it can accurately reflect the typical biochemical and morphological characteristics of apoptosis cells. In conclusion, this study demonstrated that NOB pretreatment alleviated renal I/R injury by attenuating ERS-associated apoptosis, which was dependent on the PI3K/AKT pathway. However, several other signaling pathways, such as MAPK and NF- $\mathrm{BB}$ signaling are related to the pathophysiological mechanisms of I/R injury in the kidney. Whether these signaling pathways are also associated with the protective effects of NOB against renal I/R injury remains to be determined. Altogether, the present findings indicate that NOB may represent a potential therapeutic option for renal I/R injury.

\section{Acknowledgements}

Not applicable.

\section{Funding}

No funding was received.

\section{Availability of data and materials}

The datasets used and/or analyzed during the current study are available from the corresponding author on reasonable request.

\section{Authors' contributions}

BL and WZ designed the study. QD and LZ acquired and analyzed the data. WZ drafted and revised the manuscript. All authors read and approved the final manuscript.

\section{Ethics approval and consent to participate}

The present study was approved by Jingmen No. 2 People's Hospital Laboratory Animals Ethics Committee (approval no. 4237668).

\section{Patient consent for publication}

Not applicable.

\section{Competing interests}

The authors declare that they have no competing interests.

\section{References}

1. Aboutaleb N, Jamali H, Abolhasani M and Pazoki TH: Lavender oil (lavandula angustifolia) attenuates renal ischemia/reperfusion injury in rats through suppression of inflammation, oxidative stress and apoptosis. Biomed Pharmacother 110: 9-19, 2019.

2. Gong DJ, Wang L, Yang YY, Zhang JJ and Liu XH: Diabetes aggravates renal ischemia and reperfusion injury in rats by exacerbating oxidative stress, inflammation, and apoptosis. Ren Fail 41: 750-761, 2019.

3. Kar F, Hacioglu C, Senturk H, Donmez DB and Kanbak G: The role of oxidative stress, renal inflammation, and apoptosis in post ischemic reperfusion injury of kidney tissue: The protective effect of dose-dependent boric acid administration. Biol Trace Elem Res 195: 150-158, 2019.

4. Xu Z, Zhao K, Han P, Qi X, Zhang W and Niu T: Octreotide ameliorates renal ischemia/reperfusion injury via antioxidation and anti-inflammation. Transplant Proc 49: 1916-1922, 2017. 
5. Hardi P, Nagy T, Fazekas G, Arató E, Menyhei G, Sétáló G Jr, Vecsernyés M, Pintér O, Takács I, Bohonyi N and Jancsó G: Sodium pentosan polysulfate reduced renal ischemiareperfusion-induced oxidative stress and inflammatory responses in an experimental animal model. J Vasc Res 53: 230-242, 2016.

6. Liu F, Zhang H, Li Y and Lu X: Nobiletin suppresses oxidative stress and apoptosis in $\mathrm{H} 9 \mathrm{c} 2$ cardiomyocytes following hypoxia/reoxygenation injury. Eur J Pharmacol 854: 48-53, 2019.

7. Wang T, Wang F, Yu L and Li Z: Nobiletin alleviates cerebral ischemic-reperfusion injury via MAPK signaling pathway. Am J Transl Res 11: 5967-5977, 2019.

8. Zheng Y, Bu J, Yu L, Chen J and Liu H: Nobiletin improves propofol-induced neuroprotection via regulating Akt $/ \mathrm{mTOR}$ and TLR $4 / \mathrm{NF}-\kappa \mathrm{B}$ signaling in ischemic brain injury in rats. Biomed Pharmacother 91: 494-503, 2017.

9. Liu L, Xu L, Zhang S, Wang D, Dong G, Chen H, Li X, Shu C and Wang R: STF-083010, an inhibitor of XBP1 splicing, attenuates acute renal failure in rats by suppressing endoplasmic reticulum stress-induced apoptosis and inflammation. Exp Anim 67: 373-382, 2018.

10. Liu C, Li H, Zheng H, Zhai M, Lu F, Dong S, Fang T and Zhang W: CaSR activates PKC $\delta$ to induce cardiomyocyte apoptosis via ER stress-associated apoptotic pathways during ischemia/reperfusion. Int J Mol Med 44: 1117-1126, 2019.

11. Chi X, Jiang Y, Chen Y, Yang F, Cai Q, Pan F, Lv L and Zhang X: Suppression of microRNA27a protects against liver ischemia/reperfusion injury by targeting PPAR $\gamma$ and inhibiting endoplasmic reticulum stress. Mol Med Rep 20: 4003-4012 2019.

12. Yan B, Liu S, Li X, Zhong $Y$, Tong $F$ and Yang S: Preconditioning with endoplasmic reticulum stress alleviated heart ischemia/reperfusion injury via modulating IRE1/ATF6/RACK1/PERK and PGC-1 $\alpha$ in diabetes mellitus. Biomed Pharmacother 118: 109407, 2019.

13. Chen Y, Liu S and Chen G: Aggravation of cerebral ischemia/reperfusion injury by peroxisome proliferator-activated receptor-gamma deficiency via endoplasmic reticulum stress. Med Sci Monit 25: 7518-7526, 2019.

14. Liu H, Wang L, Weng X, Chen H, Du Y, Diao C, Chen Z and Liu X: Inhibition of Brd4 alleviates renal ischemia/reperfusion injury-induced apoptosis and endoplasmic reticulum stress by blocking FoxO4-mediated oxidative stress. Redox Biol 24 101195,2019

15. Gong Z, Wang Y and Gai Y: Effects of MiR-21 on proliferation and apoptosis of fibroblast-like synoviocytes in rheumatoid arthritis through PTEN/PI3K/AKT signaling pathway. Panminerva Med Oct 24, 2019 (Online ahead of print).

16. Li C, Zhan Y, M X, Fang H and Gai X: B7-H4 facilitates proliferation and metastasis of colorectal carcinoma cell through PI3K/Akt/mTOR signaling pathway. Clin Exp Med 20: 79-86, 2020.

17. Sun W, Hu S, Hu J, Yang S, Hu B, Qiu J, Gan X, Liu H, Li L and Wang J: miR-365 inhibits duck myoblast proliferation by targeting IGF-via PI3K/Akt pathway. Biosci Rep 39: BSR20190295, 2019.

18. Amini-Khoei H, Saghaei E, Mobini GR, SabzevaryGhahfarokhi M, Ahmadi R, Bagheri N and Mokhtari T: Possible involvement of PI3K/AKT/mTOR signaling pathway in the protective effect of selegiline (deprenyl) against memory impairment following ischemia reperfusion in rat. Neuropeptides 77 : 101942, 2019.

19. Li TF, Ma J, Han XW, Jia YX, Yuan HF, Shui SF, Guo D and Yan L: Chrysin ameliorates cerebral ischemia/reperfusion (I/R) injury in rats by regulating the $\mathrm{PI} 3 \mathrm{~K} / \mathrm{Akt} / \mathrm{mTOR}$ pathway. Neurochem Int 129: 104496, 2019.

20. Liao Y, Li H, Pi Y, Li Z and Jin S: Cardioprotective effect of IGF-1 against myocardial ischemia/reperfusion injury through activation of PI3K/Akt pathway in rats in vivo. J Int Med Res 47: 3886-3897, 2019.

21. Tian Z, Tang C and Wang Z: Neuroprotective effect of ginkgetin in experimental cerebral ischemia/reperfusion via apoptosis inhibition and PI3K/Akt/mTOR signaling pathway activation J Cell Biochem 120: 18487-18495, 2019.

22. Yuan L, Fan L, Li Q, Cui W, Wang X and Zhang Z: Inhibition of miR-181b-5p protects cardiomyocytes against ischemia/reperfusion injury by targeting AKT3 and PI3KR3. J Cell Biochem 120: 19647-19659, 2019.

23. Wei Q, Zhao J, Zhou X, Yu L, Liu Z and Chang Y: Propofol can suppress renal ischemia-reperfusion injury through the activation of PI3K/AKT/mTOR signal pathway. Gene 708: 14-20, 2019.
24. Dominguez JH, Liu Y, Gao H, Dominguez JM II, Xie D and Kelly KJ: Renal tubular cell-derived extracellular vesicles accelerate the recovery of established renal ischemia reperfusion injury. J Am Soc Nephrol 28: 3533-3544, 2017.

25. Tang C, Hub Y, Gaoc J, Jiangd J, Shib S, Wanga J, Genga Q, Liange $X$ and Chaia $X$ : Dexmedetomidine pretreatment attenuates myocardial ischemia reperfusion induced acute kidney injury and endoplasmic reticulum stress in human and rat. Life Sci 257: 118004, 2020.

26. Zhang BF, Jiang H, Chen J, Guo X, Li Y, Hu Q and Yang S: Nobiletin ameliorates myocardial ischemia and reperfusion injury by attenuating endoplasmic reticulum stress-associated apoptosis through regulation of the PI3K/AKT signal pathway. Int Immunopharmacol 73: 98-107, 2019.

27. Ardic S, Gumrukcu A, Cekic OG, Erdem M, Reis Kose GD, Demir S, Kose B, Yulug E, Mentese A and Turedi S: The value of endoplasmic reticulum stress markers (GRP78 and CHOP) in the diagnosis of acute mesenteric ischemia. Am J Emerg Med 37: 596-602, 2019

28. Guoa Y, Guoa R, Sua Y,Fua J, Wanga S, Konga Y, Wua C, Wanga J, Tanc C, Mod C and Zhaoa B: The PERK/eIF2 $\alpha /$ ATF4/CHOP pathway plays a role in regulating monocrotaline-induced endoplasmic reticulum stress in rat liver. Res Vet Sci 130: 237-239, 2020.

29. Hana F, Gaoa Y, Dinga C, Xiaa X, Wanga Y, Xuea W, Dinga X, Zhenga $J$ and Tiana P: Knockdown of NLRC5 attenuates renal I/R injury in vitro through the activation of PI3K/Akt signaling pathway. Biomed Pharmacothe 103: 222-227, 2018.

30. Park WS, Park MS, Kang SW, Jin SA, Jeon Y, Hwang J and Kim SK: Hesperidin shows protective effects on renal function in ischemia-induced acute kidney injury (sprague-dawley rats). Transplant Proc 51: 2838-2841, 2019.

31. Wang Y, Peng C, Zhang Z, Shi J, Lin Y, Gu L, Ma X and Li H: Intravenous infusion of ulinastatin attenuates acute kidney injury after cold ischemia/reperfusion. Int Urol Nephrol 51: 1873-1881, 2019.

32. Wei D, Zhang G, Zhu Z, Zheng Y, Yan F, Pan C, Wang Z, Li X, Wang F, Meng P, et al: Nobiletin inhibits cell viability via the SRC/AKT/STAT3/YY1AP1 pathway in human renal carcinoma cells. Front Pharmacol 10: 690, 2019.

33. Malik S, Bhatia J, Suchal K, Gamad N, Dinda AK, Gupta YK and Arya DS: Nobiletin ameliorates cisplatin-induced acute kidney injury due to its anti-oxidant, anti-inflammatory and anti-apoptotic effects. Exp Toxicol Pathol 67: 427-433, 2015.

34. Guo J, Cui Y, Liu Q, Yang Y, Li Y, Weng L, Tang B, Jin P, Li XJ, Yang S and Li S: Piperine ameliorates SCA17 neuropathology by reducing ER stress. Mol Neurodegener 13: 4, 2018.

35. Hammad AS, Ravindran S, Khalil A and Munusamy S: Structure-activity relationship of piperine and its synthetic amide analogs for therapeutic potential to prevent experimentally induced ER stress in vitro. Cell Stress Chaperones 22: 417-428, 2017.

36. Zeng G,Lian C, Yang P,Zheng M, Ren H and Wang H:E3-ubiquitin ligase TRIM6 aggravates myocardial ischemia/reperfusion injury via promoting STAT1-dependent cardiomyocyte apoptosis. Aging (Albany NY) 11: 3536-3550, 2019.

37. Ruan Z, Wang S, Yu W and Deng F: LncRNA MALAT1 aggravates inflammation response through regulating PTGS2 by targeting miR-26b in myocardial ischemia-reperfusion injury. Int J Cardiol 288: 122, 2019.

38. Zhu R, Wang W and Yang S: Cryptotanshinone inhibits hypoxia/reoxygenation-induced oxidative stress and apoptosis in renal tubular epithelial cells. J Cell Biochem 120: 13354-13360, 2019.

39. Ding C, Han F, Xiang H, Wang Y, Dou M, Xia X, Li Y, Zheng J, Ding X, Xue W and Tian P: Role of prostaglandin E2 receptor 4 in the modulation of apoptosis and mitophagy during ischemia/reperfusion injury in the kidney. Mol Med Rep 20: 3337-3346, 2019.

40. Park SH, Gong JH, Choi YJ, Kang MK, Kim YH and Kang YH: Kaempferol inhibits endoplasmic reticulum stress-associated mucus hypersecretion in airway epithelial cells and ovalbuminsensitized mice. PLoS One 10: e143526, 2015.

41. Shu Z, Yang Y, Yang L, Jiang H, Yu X and Wang Y: Cardioprotective effects of dihydroquercetin against ischemia reperfusion injury by inhibiting oxidative stress and endoplasmic reticulum stress-induced apoptosis via the PI3K/Akt pathway. Food Funct 10: 203-215, 2019. 
42. Shen D, Chen R, Zhang L, Rao Z, Ruan Y, Li L, Chu M and Zhang Y: Sulodexide attenuates endoplasmic reticulum stress induced by myocardial ischaemia/reperfusion by activating the PI3K/Akt pathway. J Cell Mol Med 23: 5063-507, 2019.

43. Tian X, Ji Y, Liang Y, Zhang J, Guan L and Wang C: LINC00520 targeting miR-27b-3p regulates OSMR expression level to promote acute kidney injury development through the PI3K/AKT signaling pathway. J Cell Physiol 234: 14221-14233, 2019.

44. Xu Y, Wang W, Jin K, Zhu Q, Lin H, Xie M and Wang D: Perillyl alcohol protects human renal tubular epithelial cells from hypoxia/reoxygenation injury via inhibition of ROS, endoplasmic reticulum stress and activation of PI3K/Akt/eNOS pathway. Biomed Pharmacother 95: 662-669, 2017.
45. Li ZR, Yang L, Zhen J, Zhao Y and Lu ZN: Nobiletin protects PC12 cells from ERS-induced apoptosis in OGD/R injury via activation of the PI3K/AKT pathway. Exp Ther Med 16: 1470-1476, 2018.

46. Shi MD, Liao YC, Shih YW and Tsai LY: Nobiletin attenuates metastasis via both ERK and PI3K/Akt pathways in HGF-treated liver cancer HepG2 cells. Phytomedicine 20: 743-752, 2013.

This work is licensed under a Creative Commons Attribution-NonCommercial-NoDerivatives 4.0 International (CC BY-NC-ND 4.0) License. 